Research Paper

\title{
PKDI Mono-Allelic Knockout Is Sufficient to Trigger Renal Cystogenesis in a Mini-Pig Model
}

\author{
Jin $\mathrm{He}^{1,2}, \llbracket$, Qiuyan $\mathrm{Li}^{1}$, Suyun Fang ${ }^{1}$, Ying Guo ${ }^{1}$, Tongxin Liu ${ }^{1}$, Jianhua $\mathrm{Ye}^{1}$, Zhengquan $\mathrm{Yu}^{1}$, Ran Zhang ${ }^{1}$, \\ Yaofeng Zhao, ${ }^{1}$ Xiaoxiang $\mathrm{Hu}^{1}$, Xueyuan $\mathrm{Bai}^{3}$, Xiangmei Chen ${ }^{3}$, Ning $\mathrm{Li}^{1,4}, \mathbb{}$ \\ 1. State Key Laboratory for Agrobiotechnology, College of Biological Sciences, China Agricultural University, Beijing, PR China \\ 2. College of Animal Science and Technology, China Agricultural University, Beijing, PR China \\ 3. Department of Nephrology, State Key Laboratory of Kidney Disease, Chinese PLA General Hospital, Beijing, PR China \\ 4. College of Animal Science and Technology, Yunnan Agricultural University, Kunming, PR China
}

\section{$\triangle$ Corresponding author: hejin47@cau.edu.cn; ninglicaulab@gmail.com}

๑ 2015 Ivyspring International Publisher. Reproduction is permitted for personal, noncommercial use, provided that the article is in whole, unmodified, and properly cited. See http://ivyspring.com/terms for terms and conditions.

Received: 2014.10.20; Accepted: 2014.12.03; Published: 2015.02.10

\begin{abstract}
PKDI and PKD2 mutations could lead to autosomal dominant polycystic kidney disease (ADPKD), which afflicts millions of people worldwide. Due to the marked differences in the lifespan, size, anatomy, and physiology from humans, rodent ADPKD models cannot fully mimic the disease. To obtain a large animal model that recapitulates the disease, we constructed a mini-pig model by mono-allelic knockout (KO) of PKDI using zinc finger nuclease. The mono-allelic KO pigs had lower PKDI expression than their wild-type littermates at both the transcriptional and translational levels. After approximately six months, renal cysts appeared and grew progressively in the $\mathrm{KO}$ pigs. Histological analysis showed that renal cysts were scatteredly distributed in the mutant pig kidneys and were lined by either cuboidal or flattened epithelial cells. Contrast-enhanced computed tomography confirmed that all of the mutant pigs had renal and hepatic cysts, when they were 11 -month-old. Immunohistochemical analysis revealed that most of the cysts were derived from the proximal tubules and collecting ducts. Therefore, the PKDI mono-allelic knockout is sufficient to trigger renal cystogenesis, and this pig model may provide a platform for future study of renal cyst formation.
\end{abstract}

Key words: ADPKD, PKD1, ZFN, Disease model

\section{Introduction}

Progressively enlarged fluid-filled cysts in kidneys and other organs are the key features of autosomal dominant polycystic kidney disease (ADPKD), which afflicts millions of people worldwide [1]. No effective therapeutic approach, except for renal replacement therapy, is currently available for ADPKD, a disease that ultimately leads to end stage renal disease in approximately half of the affected patients [2, 3]. Genetic studies have demonstrated that mutations in two genes, $P K D 1$ and $P K D 2$, are responsible for $\sim 85 \%$ and $\sim 15 \%$ of ADPKD cases, respectively [4-6]. In addition, because of the early onset of cystogenesis, patients with PKD1 mutations have significantly more severe disease than those with mutant PKD2 [7]. Although numerous mutations have been found to be associated with ADPKD thus far, no evident mutation hotspots have been identified in either of the two genes, obscuring our understanding of the genetic basis of ADPKD. Therefore, various hypotheses have been proposed to explain the cyst formation, including two-hit, third hit, hypomorphic/hypermorphic effects, and haploinsufficiency, based on which rodent models have been generated to study the etiology of ADPKD [8]. Much information has been obtained using these models thus far, such as the identification of the signaling pathways involved in 
ADPKD; additionally, drugs have been tested and screened. However, the short lifespan, fast disease progression, dissimilarities in anatomy/physiology to humans, and late/rare cyst formation in mono-allelic PKD1 or PKD2 knockout $(\mathrm{KO})$ rodent models have hindered the utility of rodents as a model for this chronic disease $[9,10]$.

Pigs, which are highly similar to humans in their anatomy, physiology, and genetics, are becoming a more attractive animal model for biomedical research [11]. A number of genetically modified porcine models have been reported for cystic fibrosis [12], neurodegenerative disorder [13], and xenotransplantation [14]. However, due to the lack of germ-line competent embryonic stem cells, it is very inefficient to produce KO pigs using conventional gene-targeting methods. With the advent of genome editing tools, such as zinc finger nuclease (ZFN), transcription activator-like effector nuclease (TALEN), and clustered, regularly interspersed, short panlindromic repeats (CRISPR)/ CRISPR-associated (Cas) protein cas9, highly efficient production of $\mathrm{KO}$ pigs has become a reality [15-17].

In previous studies, we cloned and characterized the pig PKD1 and PKD2 genes and demonstrated a remarkable similarity between the human and pig orthologs at molecular level $[18,19]$. Furthermore, a porcine PKD2 transgenic model was generated, but there were no cystic manifestations by one year of age in that model [20]. To obtain a relatively early-onset disease model and to document whether the mono-allelic $\mathrm{KO}$ of PKD1 (PKD1+-) triggers renal cyst formation, we utilized ZFN technology to generate $\mathrm{PKD}^{+/}$Chinese experimental mini-pigs (CEMP), which showed macroscopically visible renal cysts within one year.

\section{Materials and methods}

\section{ZFN.}

ZFN was designed by Sigma-Aldrich. Different amounts of ZFN were used for optimization of transfection. Twenty-four hours after transfection, the fibroblasts were subjected to DNA extraction (Qiagen). Then the ZFN-F (TAATTACAGGGGCCAAGCAG) and ZFN-R (GAGGCAGGGAAGACGTTGT) primers were designed to sequence the target site, and ZFN-mediated gene editing efficiency was calculated according to one hundred TA-clone sequencing results. The optimized transfection conditions resulted in high gene editing efficiency and low cell toxicity. Based on the standard, transfection with $0.5 \mu \mathrm{g}$ of each ZFN plasmid was used for subsequent study.

\section{Chinese experimental mini-pig.}

Chinese experimental mini-pig (CEMP) was established by full-sib inbreeding and negative selection of Xiang pigs [21]. CEMP, which has a life-span of 16-18 years, reaches sexual maturity at 4-6 months old. The sow can deliver $7 \sim 8$ piglets per litter with a gestation period of 114 days. The neonatal pigs weigh about $0.4-0.5 \mathrm{~kg}$, and then grow to $5 \mathrm{~kg}$ at 2-month-old. When they are 6-month-old, the body weight is about $20 \mathrm{~kg}$. The adult pigs weigh approximately $40-50 \mathrm{~kg}$ with a height of $45-50 \mathrm{~cm}$ at one-year-old. The kidney, liver, heart, and stomach weight-to-body weight ratio of CEMP are similar to human being's, which makes it an intriguing model for biomedical research [21].

\section{Generation of cloned pigs.}

CEMP embryonic fibroblasts were obtained from an E30 day male embryo and were cultured in DMEM (Sigma) with $10 \%$ fetal bovine serum (Gibco) at $37^{\circ} \mathrm{C}$. A neomycin-resistant cassette was derived from the pL452 plasmid by EcoRI and BamHI digestion. A total of $1.5 \mu \mathrm{g}$ DNA $(0.5 \mu \mathrm{g}$ for each ZFN construct and 0.5 $\mu \mathrm{g}$ of neomycin-resistant cassette) was transfected into $1 \times 10^{6}$ fibroblasts. Subsequent procedures, including the screening of the cells, colony formation, transfer, and conservation were similar to a previous report [20]. Genotyping of the 72 surviving colonies was performed using the ZFN-F/R primers. Potential off-target sites were sequenced using eight pairs of primers (listed in Supplementary Material: Table S1) flanking these sites. After ruling out off-target events, four fibroblast colonies (Nos. 60, 63, 77, and 96) were selected for somatic cell nuclear transfer [22]. Thirteen surrogate sows each received an average of 384 reconstructed embryos. After approximately 114 days, 20 piglets were delivered naturally from five sows. Genotyping was performed immediately using the ZFN-F/R primers and DNA extracted from ear biopsies. The cloned pigs were fed the same food and water.

\section{PKDI expression analysis.}

Quantitative real-time PCR (qRT-PCR) was employed to detect $P K D 1$ expression in $\mathrm{WT}$ and $P K D 1^{+-}$ pigs. RNA was extracted from ear-biopsies and kidneys of the cloned pigs by RNeasy (Qiagen) and RNase-free DNase (Qiagen). The primers for PKD1 3' part were 3'-Q-F (CATGTGGCTCCTCTCAAGCA) and 3'-Q-R (GCTTCCAGCAGGACCTTGAGT) targeting exons $36 \& 37$, for $5^{\prime}$ part were $5^{\prime}-\mathrm{Q}-\mathrm{F}$ (ACGTCGGGCTCCTAGAGAA) and 5'-Q-R (TTCCCGCTCAGGTTTATTTC) targeting exons 2 \& 3 , while those for the internal control, GAPDH, were GAPDH-F (ATCACCATCTTCCAGGAGCGA) and GADPH-R (AGCCTTCTCCATGGTCGTGAA). One microgram of RNA from these tissues was reverse-transcribed into cDNA using oligo dT or ran- 
dom primer in a volume of $25 \mu \mathrm{L}$. Then, qRT-PCR reactions were performed in triplicate.

Membrane protein was extracted from the kidneys using the Membrane and Cytosol Protein Extraction Kit (Beyotime, Nantong, China). Eighty micrograms of membrane protein per lane was separated, transferred, and blotted according to a previously established protocol [18]. The antibodies used to detect PC1 were 7e12 (gift from Dr. Christopher Ward) with 1:500 dilution. For internal control, the mouse monoclonal anti- $\mathrm{Na}^{+}, \mathrm{K}^{+}$-ATPase antibody (Abcam) was utilized at a 1:1,000 dilution.

\section{Histological analysis.}

Kidneys from cloned pigs were harvested, minced, and fixed in 10\% neutral buffered formalin solution. Then, the samples were dehydrated and embedded in paraffin. For HE analysis, 5 - $\mu$ m-thick sections were stained with hematoxylin and eosin. Antibodies for a-SMA (1:100, Abcam, Shanghai, China), Lrp2 (1:100, Abcam), THP (1:100, Santa Cruz), Calbindin-D-28K (1:100, Sigma-Aldrich), and biotinylated-DBA (1:200, Vector Laboratories, CA) were used for immunohistochemistry by the $A B C$ method (Vector Laboratories). Cyst index, which was calculated using ImageJ (NIH, Bethesda, MD) as the area of lumen divided by the section area, was performed on the WT kidney sections and the non-cystic regions of mutant kidneys.

\section{Contrast enhanced computed tomography.}

After anesthetization, pigs were injected with Ultravist (Bayer) as contrast medium. Then, CT was performed with a multi-splice spiral CT instrument (Lightspeed Plus, GE), with the following parameters: $120 \mathrm{kV}, 500 \mathrm{~ms}$, and $1.5 \mathrm{~mm}$ splice thickness. The adjusted renal volume was presented as the mean value of the left and right kidneys normalized to the body weight. CT was performed in the radiology department of either Zhuozhou Chinese Medical Hospital or PLA General Hospital. The renal/hepatic cyst identification and $3 \mathrm{D}$ reconstruction were conducted by experienced radiologists from the Department of Radiology, PLA General Hospital.

\section{Statistical analysis.}

Data are presented as either mean \pm SEM or mean \pm SD. The unpaired student's $t$ test was used to compare the difference between the WT pigs and each $P K D 1^{+/}$genotype. The paired student's $t$ test was employed to determine changes in the cyst diameter. $\mathrm{P}<0.05$ was considered statistically significant.

\section{Ethical Statement.}

All of the procedures were conducted according to the guidelines developed by the China Council on
Animal Care and Protocol and were approved by China Agricultural University (No. SKLAB-201204-03).

\section{Results and discussion}

ZFN was designed to target exon 5 of PKD1, where ZFN-induced nucleotide insertions and deletions (indels) are expected to cause frame-shifting, and disrupt normal gene expression (Fig. 1A). After optimization of the transfection conditions (Supplementary Material: Table S2), ZFN plasmids, together with a neomycin-resistant cassette, were then delivered into CEMP fibroblasts obtained from a male embryo (Supplementary Material: Fig. S1A). After seven days of selection with G418, 72 colonies survived. PCR was performed to examine the genotypes of these cells, and 12 colonies $(16.7 \%)$ were found to harbor mutant PKD1 alleles, of which three colonies contained compound heterozygous $\mathrm{KO}$ events $(4.2 \%)$ (Fig. 1B). Our results showed a higher efficiency than previous reports, which generated heterozygous and homozygous KO fibroblasts with efficiencies of $4.2 \%$ and $1 \%$, respectively $[15,16]$. To select colonies without off-target modifications of the genome, eight most likely potential off-target sites, each containing six mismatched nucleotides to the ZFN target, were sequenced for all 12 mutant colonies. These potential off-target sites were screened based on the released pig genome sequence [23]. No mutations were identified in the off-target sites, proving high specificity of the ZFN used in our experiment (Supplementary Material: Table S1). Mono-allelic KO colonies Nos. 60, 63, and $96\left(\mathrm{PKD}^{+/}\right)$and a compound heterozygous allele-harboring colony No. $77\left(P K D 1^{-/}\right)$were then selected as donors for somatic cell nuclear transfer (SCNT). A total of 4,987 reconstructed embryos were transferred to 13 surrogate sows, and 20 healthy piglets were delivered naturally from five recipients (Supplementary Material: Table S3). Sequencing was conducted to confirm the genotypes of these cloned pigs, of which seven piglets had a TGCT insertion allele (PKD1 ${ }^{\text {TGCT }}$ ins/+; c.642_643insTGCT, p.Phe215Lysfs ${ }^{*} 197$ ), while six piglets had a $\mathrm{T}$ insertion allele (PKD1T ins/+; c.642_643insT, p.Ala216Cysfs $\left.{ }^{*} 195\right)$ (Fig. 1C). The two frame-shifted alleles with the appearance of TGA at $1,447-1,449 \mathrm{bp}$ or $1,450-1,452 \mathrm{bp}$ of the PKD1 mRNA (GenBank ID: NM_001246202) encoding two 2-residue-difference truncated polypeptides $(\sim 42 \mathrm{kDa})$ that only preserved signal peptide and LRR domains of Polycystin-1 (PC1) (Supplementary Material: Fig. S1B). Moreover, no piglets derived from colony No. 77 were born in our experiment, perhaps because colony No. 77 was incapable of SCNT, or the $P K D 1^{--}$resulted in embryonic lethality. As in mouse models that disrupted either PKD1 or 
$P K D 2$, prenatal and perinatal deaths were common phenomena, which demonstrates that both genes are required for normal placental development [24]. Because PKD1 is ubiquitously expressed in various organs [18], qRT-PCR was performed using RNA from ear biopsies of these piglets. As shown in Fig. 1D and Supplementary Material: Fig. S1C, $P K D 1^{+/}\left(P K D 1^{\text {TGCT }}\right.$ ins/+ and $P K D 1^{\text {Tins } /+}$ ) pigs showed a nearly $40 \%$ reduction in PKD1 expression, proving that the majority of the mutant alleles cannot produce full length mRNA. To confirm decreased $P K D 1$ expression in $P K D 1^{+/-}$pig kidneys, one neonatal pig (48h) with each genotype was randomly chosen and sacrificed. RNA and membrane protein extracted from the kidneys were subjected to qRT-PCR and Western blot analysis, re-

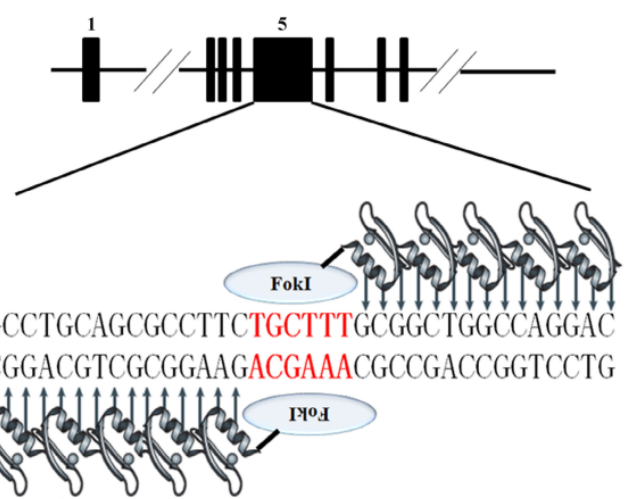

C

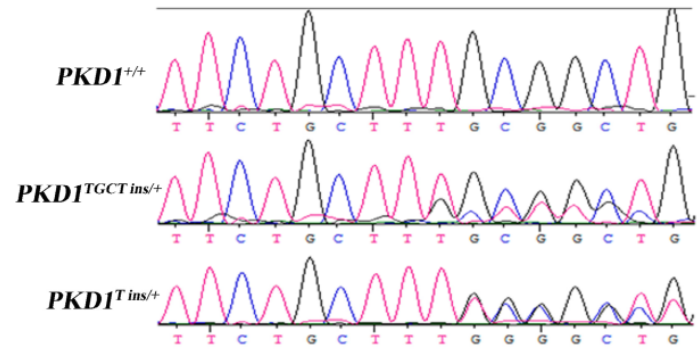

wT

No.9

No. 13

No. 22

No. 32

No. 49

No. 60

No. 63

No. 69

No. 75

No. 77

No. 93 No. 96 spectively. As shown in Figs. $1 \mathrm{E}$ and $\mathrm{F}$ and Supplementary Material: Fig. S1D, PKD1 expression in KO pigs were lower than in $P K D 1^{+/+}$pig at both the transcriptional and translational levels. Additionally, we detected additional bands with similar molecular weight to the truncated $\mathrm{PC} 1$ in $P K D 1^{+/}$pigs (Fig. $1 \mathrm{~F}$ ), which meant that mutant alleles could generate truncated PC1 to some extent. At sexual maturity, some of the $P K D 1^{+-}$pigs were mated with WT sows, and a total of 64 offspring were secured, of which 35 piglets inherited the mutant alleles (Supplementary Material: Table S4). This result demonstrated that the mutant alleles could be passed through germline transmission.

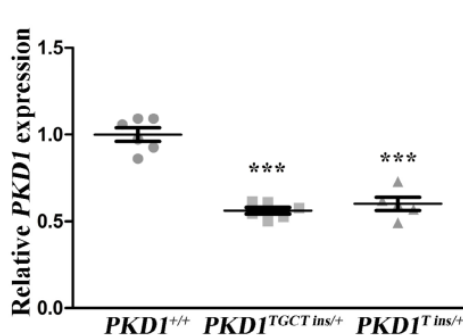

$\mathbf{E}$

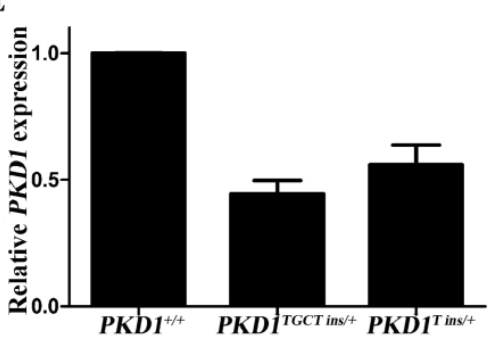

F

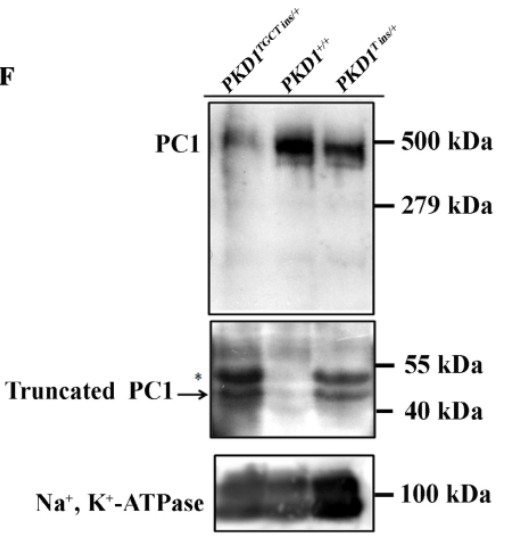

Figure 1. Generation and molecular characterization of PKDI+/- pigs. (A) Schematic representation of the ZFN targeting site of PKDI. The red letters indicate the ZFN cleavage site, and the black letters are the ZFN binding sequence. (B) The genotypes of the 12 mutant colonies, of which Nos. 13, 22, and 77 were compound heterozygous colonies. $\Delta$ and + indicate the deletion and insertion of nucleotides, respectively, compared to the WT allele. The underlined red letters represents the ZFN binding sequence. The lowercase letters highlighted in yellow denote the inserted nucleotides, and dots mean the deleted nucleotides. (C) Representative direct sequencing results of the WT, TGCT insertion and Tinsertion alleles, respectively. (D) qRT-PCR analysis of PKDI expression (mean \pm SEM) in neonatal pigs, showing that PKD I TGCT insl+ $(n=6)$ and PKDIT ins/+ $(n=5)$ pigs had reduced expression compared to WT pigs $(n=6$, **** $P<0.001)$. (E) qRT-PCR of PKDI expression in neonatal kidneys showing a reduction of renal PKDI in PKDI+/- pigs. Data obtained from three replicated experiments are presented as the mean \pm SD. Primers amplifying $3^{\prime}$ part of $P K D I$ were used in (D) and (E). (F) The PCl levels of these cloned pigs were measured by Western blotting of membrane protein extracted from neonatal kidneys using 7e12 antibody. The full-length of $\mathrm{PCl}$ is shown in the upper panel in all pigs (exposure time: $10 \mathrm{~min}$ ). The arrow in the middle panel indicates the truncated PCl produced by mutant PKDI alleles, while the asterisk indicates an $\sim 50 \mathrm{kDa}$ band that might be the glycosylated form of the truncated PCl (exposure time: $30 \mathrm{~min}$ ). The PKDI+/+ pig does not have the truncated PCl fragment. $\mathrm{Na}^{+}, \mathrm{K}^{+}-\mathrm{ATPase}$ was used as an internal control (exposure time: 5 min). The three panels are from three different blots, but they are running simultaneously with the same load of protein. 

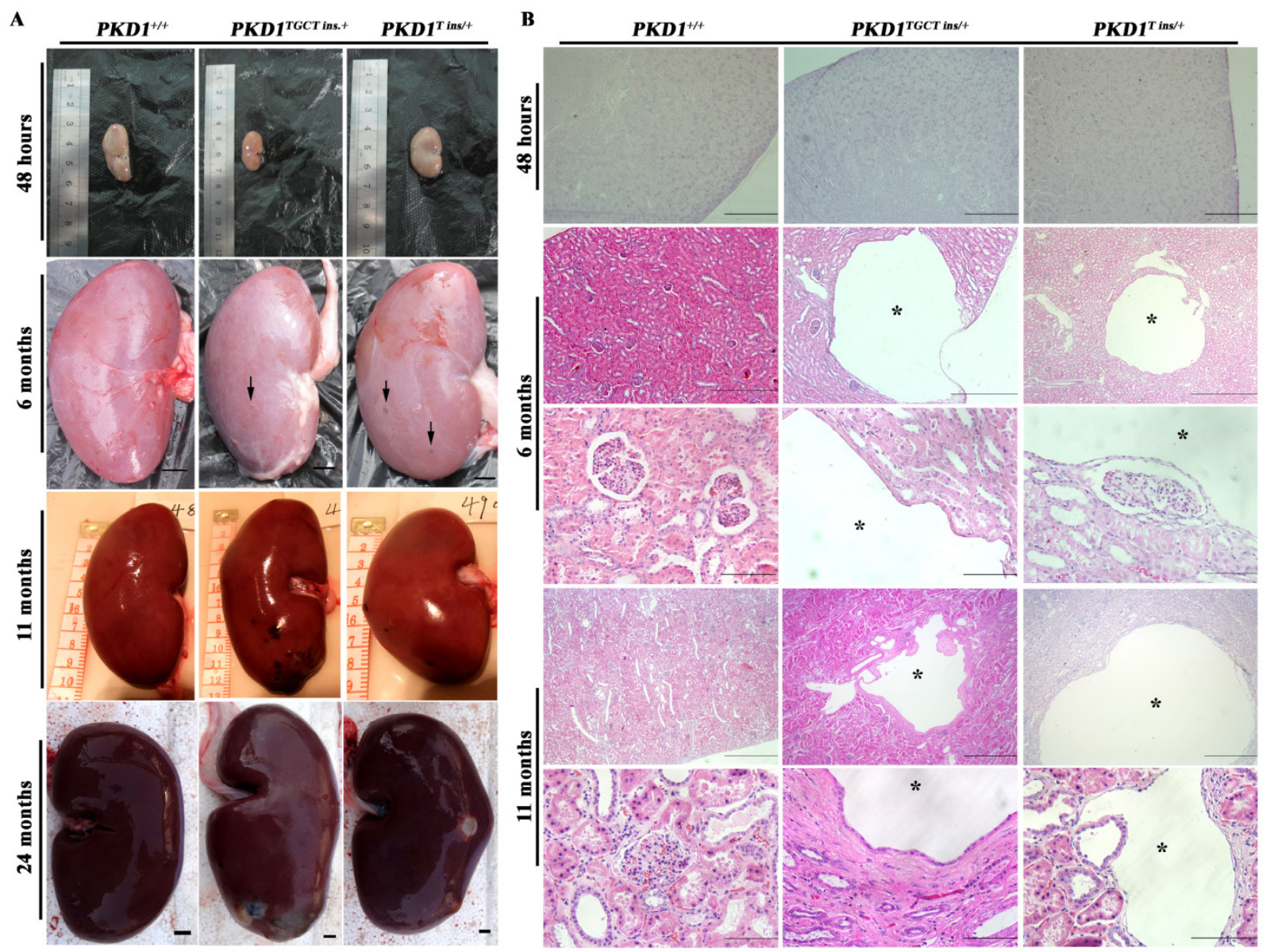

Figure 2. PKDI+/- pigs show the typical ADPKD phenotype. (A) The gross morphology of the kidneys collected at different ages exhibits macroscopic cysts (arrow) on the surface of the PKDI+/- pig kidneys (scale bar $=1 \mathrm{~cm}$ ). (B) Hematoxylin and eosin analysis of the kidneys reveals typical renal cysts $(*)$ lined by either flattened or cuboidal epithelia in the PKDI+/- pigs (scale bar for neonatal kidneys is $1 \mathrm{~mm}$; for upper panel of 6-month-old kidneys, the bar is 500 um; for the lower panel of 6-month-old kidneys, it is $100 \mu \mathrm{m}$; for the upper panel of 11 -month-old kidneys, it is $1 \mathrm{~mm}$; for the lower panel of 11 -month-old kidneys, it is $100 \mu \mathrm{m}$ ). For each stage, at least 30 renal sections or 20 cysts were analyzed.

No macroscopic or microscopic cysts had developed in the $P K D 1^{+/}$pig kidneys by 48 hours after birth, suggesting that half the amount of normal PC1 might be sufficient for renal development (Fig. 2). To investigate whether there are pathological changes in 6-month-old $P K D 1^{+/}$pig kidneys, one pig from each of the three groups was sacrificed, and the kidneys were collected and fixed. Some small but macroscopically visible fluid-filled cysts began to emerge on the surfaces of the $\mathrm{PKD1}^{+-}$pig kidneys (Fig. 2A). The largest cysts at this stage were $\sim 5 \mathrm{~mm}$ in diameter. At 11 months old, the cysts were growing larger and the number increased, though the cysts were still scattered throughout the $P K D 1^{+/}$kidneys. In the cystic kidneys, both flattened and cuboidal epithelial cells were found in the lining of the renal cysts (Fig. 2B and Supplementary Material: Fig. S2A). At 24 months, these cysts began to slightly deform the normal kidney shape (Fig. 2A). Further analysis also confirmed the appearance of renal cysts in $\mathrm{F} 1 \mathrm{PKD} 1^{+/}$piglets by as early as 3 months after birth, suggesting the stable inheritance of PKD1 mutant alleles (Supplementary
Material: Fig. S2A). Additionally, we measured the cyst index of the non-cystic regions of mutant kidneys. Although we could find at least 50 cysts/microcysts in each $\mathrm{KO}$ pig kidneys, the cyst index did not show any signs of tubule dilation $(20.0 \%$ $\pm 0.8 \%)$ compared with WT kidney sections $(19.5 \% \pm$ $2.0 \%$ ) (Supplementary Material: Fig. S2B). This result suggested that the disease was in the early-stage without affecting the whole kidney.

As an accurate approach for detecting renal cysts, contrast-enhanced computed tomography (CT) was employed to examine the 11-month-old pigs. To our knowledge, there is no CT diagnostic criterion for ADPKD; thus, we adopted an ultrasonographic standard to examine these pigs [25]. In this criterion, the presence of at least 3 cysts in one or two kidneys from patients younger than 30 years of age would confirm the diagnosis of ADPKD. Because contrast-enhanced CT can detect a cyst of at least $1.5 \mathrm{~mm}$, we set the threshold to $5 \mathrm{~mm}$ for counting cysts to minimize the false-positive diagnosis. We found that many different-sized cysts were present in the bilat- 
eral kidneys of all tested $P K D 1^{+/}$pigs (Fig. 3 and Supplementary Material: Table S5). Means of nine and five cysts in bilateral kidneys of the 11-month-old pigs had larger diameters than $5 \mathrm{~mm}$ in the PKD1TGCT ins/+ and $P K D 1^{T \text { ins } /+}$ pigs, respectively. The largest diameter was $28.3 \mathrm{~mm}$ in a $P K D 1^{\text {TGCT ins/+ }}$ pig, while a $P K D 1^{\text {Tins/+ }}$ pig had a $19.3 \mathrm{~mm}$ cyst. In addition to renal cysts, liver cysts, observed in approximately $80 \%$ of ADPKD cases [26], were found in all $P K D 1^{+/}$pigs (Fig. 3).

To confirm that these cysts could grow gradually, CT was performed on 18-month-old pigs. The detected renal cysts were larger than those from 11-month-old pigs by $1.27 \pm 0.30 \mathrm{~mm} \quad(\mathrm{P}=0.0002)$ (Supplementary Material: Fig. S1E). In current studies, total kidney volume (TKV) is recognized as a more accurate marker for polycystic kidney disease, as changes in this measurement mainly reflect alterations in the cyst numbers and volumes [27]. Thus, in our study, 3D reconstruction and calculation of kidney volumes were performed, but no TKV difference was observed in the $P K D 1^{+-}$pigs at this stage compared with the WT littermates (Supplementary Material: Fig. S1F). The result meant that these mono-allelic $\mathrm{KO}$ pigs were in the early stage of disease progression without significant renal enlargement.

As human renal cysts can arise from all nephron segments and collecting ducts, segment-specific markers for nephrons and collecting ducts were used to identify the origin of the cysts in our experiment. All the markers except for THP, which stains thick ascending limbs of loop of Henle (LH) and distal tubules (DT), were positively stained (Fig. 4), indicating that the cysts derived mainly from proximal tubules (PT) and collecting ducts (CD). Our results are similar to the recent report of Hopp et al, who demonstrated in a mouse ADPKD model that cysts originating from the LH and DT were consistently $<1 \%$ [28]. Another ADPKD characteristic like renal fibrosis, which is associated with disease progression, can also be observed in the $P K D 1^{+-}$pigs [29]. Early-stage human ADPKD often have focal staining of a-SMA, similar to our result shown in Fig. 4, where a-SMA mainly surrounded the renal cysts suggesting the increased number of interstitial myofibroblasts. These cells are typical feature of fibrosis, and can participate in the deposition of extracellular matrix [29].

As young human patients only have rare cysts, which is reflected by the diagnostic criterion [25], our $P K D 1^{+/-}$pigs, which is comparable to human adolescence, shows some aspects of early-stage human ADPKD in terms of sporadic renal and hepatic cysts. Until now, mouse models, which have provided us with essential knowledge of ADPKD, are still the predominant animal models used in ADPKD research [8]. However, no effective therapies have developed thus far from these models, e.g., some drugs, which slow down disease progression in ADPKD mice, failed in preclinical trials [30,31]. The possible reason is likely due to the differences in anatomy, physiology, metabolism and genetics between mouse and human. Over the mouse models, pigs show many advantages, which may either supplement our understanding of ADPKD from a different angle or act as a bridge between clinical trial and basic research. First, porcine kidney has a comparable size and similar multipapillate structure to human kidney, making it an ideal model for histological study and sample [32]. Second, ADPKD is a systemic disease with many extrarenal manifestations [1], such as cardiovascular abnormalities, intracranial aneurysm and hypertension, some of which can be better studied using pigs than mice.

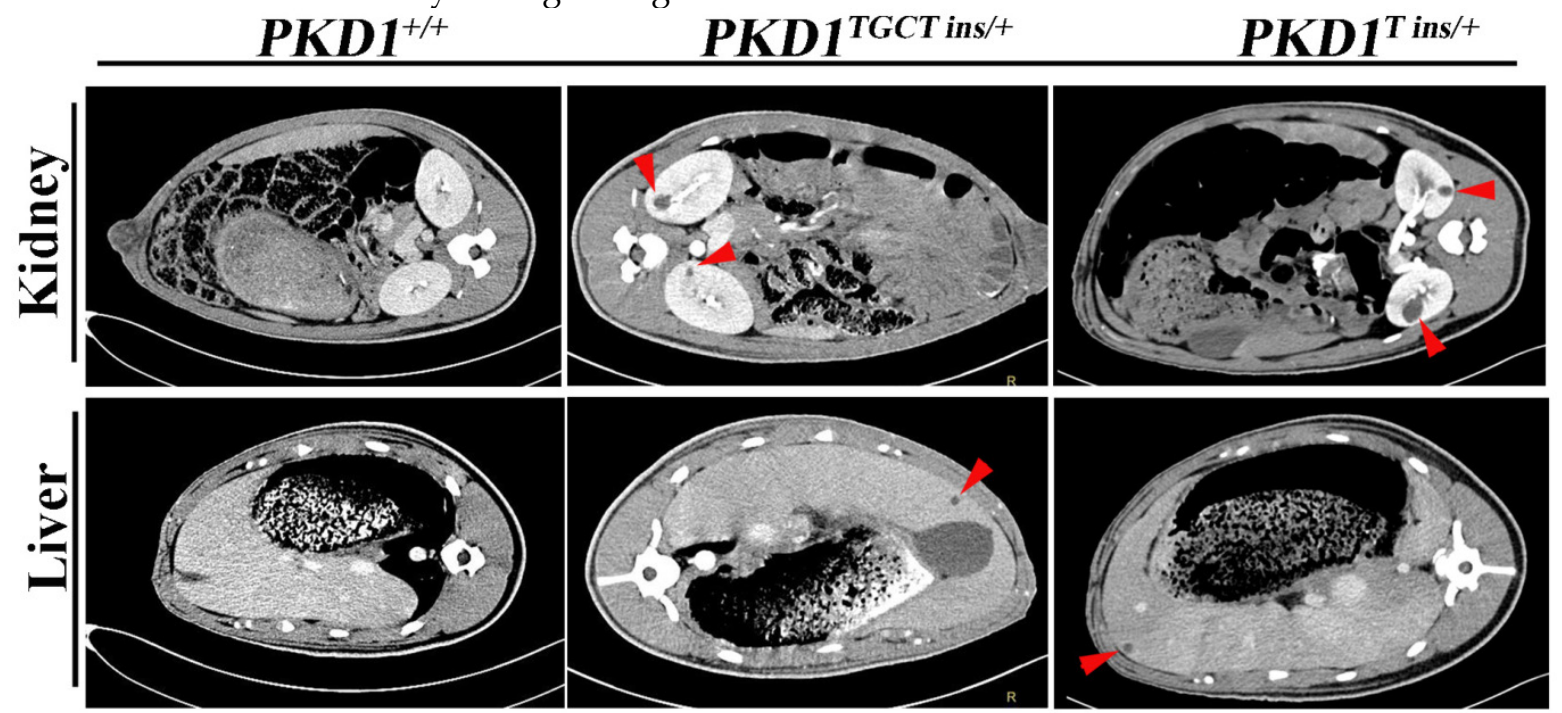

Figure 3. Contrast enhanced computed tomography was performed to diagnose the renal and hepatic cysts in PKD I+/- pigs at 11 -month-old. Red arrowheads show the typical cysts in the kidneys and livers. 

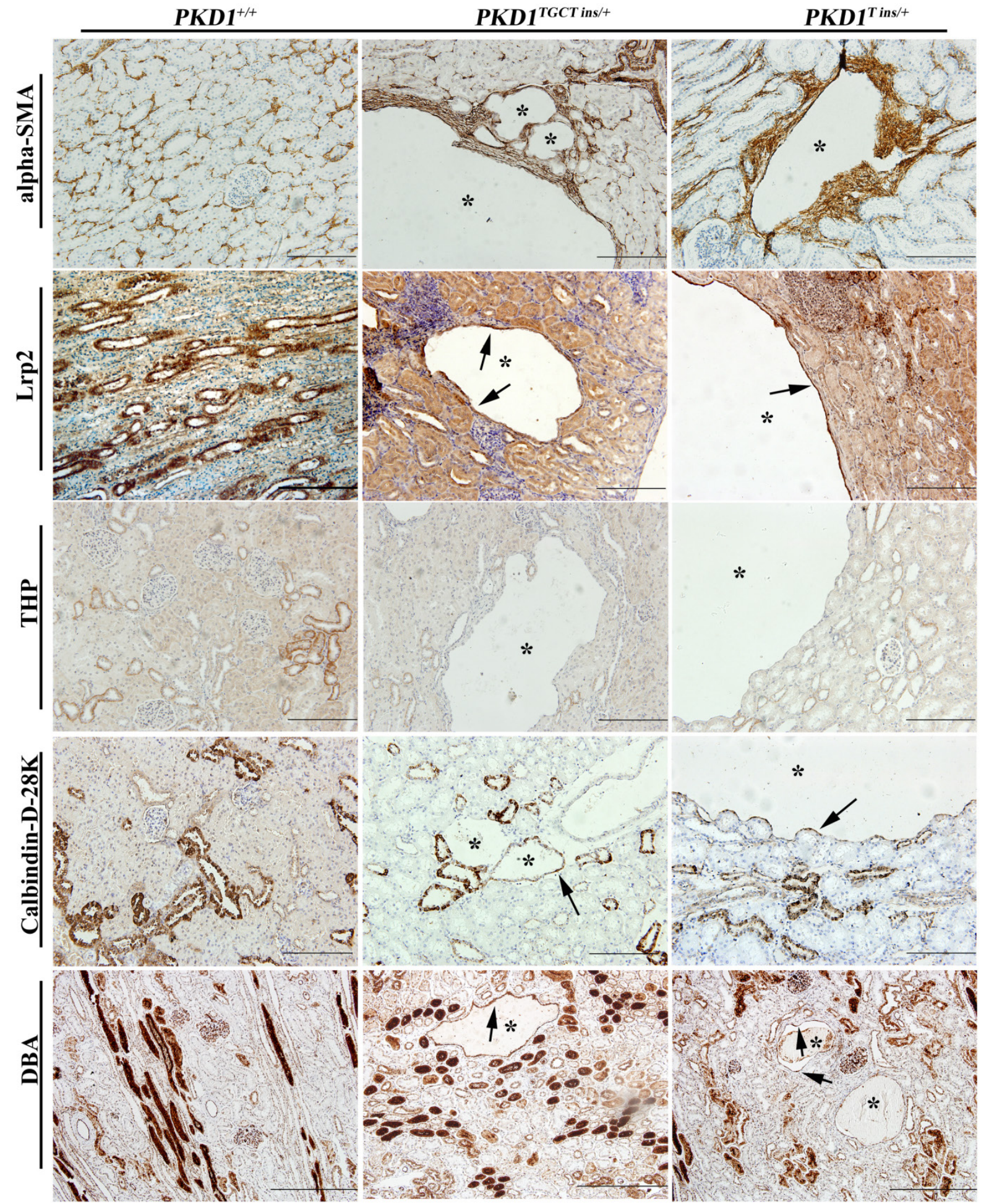

Figure 4. Immunohistochemical characterization of renal cysts in PKDI+- pigs. The upper panel shows that $\alpha$-SMA staining fibrosis surrounding renal cysts $(*)$ in the PKDI+/ pigs. The origin of renal cysts was determined using segment-specific markers/antibodies for proximal tubules (Lrp2), thick ascending limbs of loop of Henle/distal convoluted tubules (Tamm-horsefall protein, THP), distal convoluted tubules/cortical collecting ducts (Calbindin-D-28K), and collecting ducts (Dolichos Biflorus agglutinin, DBA). Cysts were positively stained by all of these antibodies/markers (arrow) except for THP. Each tubular segment specific marker was used to stain at least 30 cysts in each PKDI+l- kidneys. Scale bars in the images are $200 \mu \mathrm{m}$, except for DBA staining, which are $500 \mu \mathrm{m}$.

Third, compared to mono-allelic PKD1 or PKD2 $K O$ mice, which showed late-onset cystogenesis or no cyst formation $[9,10,33,34]$, our $P K D 1^{+/-}$pigs have a relatively early and progressed cystogenesis. Finally, as a chronic disease, our CEMP pigs, which have a lifespan of at least 16 years, could be readily used for longitudinal studies of ADPKD. Additionally, the $P K D 1^{+/-}$pigs could also be used as a platform to facil- 
itate the construction of other related models. As we showed that PKD1 mutant alleles could be passed to offspring by germline transmission (Supplementary Material: Table S4), $P K D 1^{+/}$embryonic fibroblasts can be established after the mating of $P K D 1^{+/}$and $P K D 1^{+/+}$ pigs. With modifications to the normal PKD1 allele in fibroblasts by introducing a hypomorphic mutation, a faster progressing disease model than $P K D 1^{+/}$could be obtained; or by knockout of PKD2, a pig with the $P K D 1^{+/,} P K D 2^{+/-}$genotype can be produced to elucidate the trans-heterozygosity hypothesis.

\section{Conclusion}

Our experiment demonstrated that mono-allelic KO of PKD1 could trigger renal and hepatic cysts formation. This model may be served as an alternative model for ADPKD because of the observed early-onset of cystogenesis, combined with relatively slow disease progression, mimicking the chronic nature of human ADPKD. Therefore, the model we developed could be used either to study the molecular mechanism of cystogenesis, or as a basis to construct other fast-progressed disease models.

\section{Supplementary Material}

Tables S1-S5, Figures S1-S2.

http://www.ijbs.com/v11p0361s1.pdf

\section{Abbreviations}

ADPKD, autosomal dominant polycystic kidney disease; CEMP, Chinese Experimental mini-pig; CRISPR/Cas9, clustered, regularly interspersed, short panlindromic repeats (CRISPR)/CRISPR-associated (Cas) protein cas9; CT, computed tomography; DT, distal tubule; indel, insertion and deletion; $\mathrm{KO}$, knockout; LH, loops of Henle; PC1, polycystin-1; PKD1, polycystic kidney disease 1 gene; PKD2, polycystic kidney disease 2 gene; PT, proximal tubule; SCNT, somatic cell nuclear transfer; TALEN, transcription activator like effector nuclease; TKV, total kidney volume; WT, wild type; ZFN, zinc finger nuclease.

\section{Acknowledgments}

This work was supported by National Basic Research Program of China (No. 2011CB944103) and National Natural Science Foundation of China (No. 81370840). H.J. was also sponsored by the Chinese Universities Scientific Fund (No. 2013BH002). We thank Dr. Tian Shuping (Chinese PLA General Hospital) for the CT analysis.

\section{Competing Interest}

A patent application on the PKD1 ${ }^{\mathrm{TGCT}}$ ins/+ and PKD1 ${ }^{\text {Tins/+ }}$ pigs has been submitted by N. L and J. H. as the inventors.

\section{References}

1. Gabow PA. Autosomal dominant polycystic kidney disease--more than a renal disease. Am J Kidney Dis. 1990; 16: 403-13.

2. Torres VE, Harris PC. Autosomal dominant polycystic kidney disease: the last 3 years. Kidney Int. 2009; 76: 149-68. doi:10.1038/ki.2009.128.

3. Wilson PD. Polycystic kidney disease. N Engl J Med. 2004; 350: 151-64. doi:10.1056/NEJMra022161.

4. Kimberling WJ, Kumar S, Gabow PA, Kenyon JB, Connolly CJ, Somlo S. Autosomal dominant polycystic kidney disease: localization of the second gene to chromosome 4q13-q23. Genomics. 1993; 18: 467-72.

5. Mochizuki T, Wu G, Hayashi T, Xenophontos SL, Veldhuisen B, Saris JJ, et al. PKD2, a gene for polycystic kidney disease that encodes an integral membrane protein. Science. 1996; 272: 1339-42.

6. Pignatelli PM, Pound SE, Carothers AD, Macnicol AM, Allan PL, Watson ML, et al. Multipoint mapping of adult onset polycystic kidney disease (PKD1) on chromosome 16. J Med Genet. 1992; 29: 638-41.

7. Harris PC, Bae KT, Rossetti S, Torres VE, Grantham JJ, Chapman AB, et al. Cyst number but not the rate of cystic growth is associated with the mutated gene in autosomal dominant polycystic kidney disease. J Am Soc Nephrol. 2006; 17: 3013-9. doi:10.1681/ASN.2006080835.

8. Wilson PD. Mouse models of polycystic kidney disease. Curr Top Dev Biol. 2008; 84: 311-50. doi:10.1016/S0070-2153(08)00606-6.

9. Lu W, Fan X, Basora N, Babakhanlou H, Law T, Rifai N, et al. Late onset of renal and hepatic cysts in Pkd1-targeted heterozygotes. Nat Genet. 1999; 21: 160-1. doi:10.1038/5944.

10. Chang MY, Parker E, Ibrahim S, Shortland JR, Nahas ME, Haylor JL, et al. Haploinsufficiency of $\mathrm{Pkd} 2$ is associated with increased tubular cell proliferation and interstitial fibrosis in two murine Pkd2 models. Nephrol Dial Transplant. 2006; 21: 2078-84. doi:10.1093/ndt/gfl150.

11. Aigner B, Renner S, Kessler B, Klymiuk N, Kurome M, Wunsch A, et al. Transgenic pigs as models for translational biomedical research. J Mol Med (Berl). 2010; 88: 653-64. doi:10.1007/s00109-010-0610-9.

12. Rogers CS, Stoltz DA, Meyerholz DK, Ostedgaard LS, Rokhlina T, Taft PJ, et al. Disruption of the CFTR gene produces a model of cystic fibrosis in newborn pigs. Science. 2008; 321: 1837-41. doi:10.1126/science.1163600.

13. Yang D, Wang CE, Zhao B, Li W, Ouyang Z, Liu Z, et al. Expression of Huntington's disease protein results in apoptotic neurons in the brains of cloned transgenic pigs. Hum Mol Genet. 2010; 19: 3983-94. doi:10.1093/hmg/ddq313.

14. Dai Y, Vaught TD, Boone J, Chen SH, Phelps CJ, Ball S, et al. Targeted disruption of the alpha1,3-galactosyltransferase gene in cloned pigs. Nat Biotechnol. 2002; 20: 251-5. doi:10.1038/nbt0302-251.

15. Yang D, Yang H, Li W, Zhao B, Ouyang Z, Liu Z, et al. Generation of PPARgamma mono-allelic knockout pigs via zinc-finger nucleases and nuclear transfer cloning. Cell Res. 2011; 21: 979-82. doi:10.1038/cr.2011.70.

16. Hauschild J, Petersen B, Santiago Y, Queisser AL, Carnwath JW, Lucas-Hahn $\mathrm{A}$, et al. Efficient generation of a biallelic knockout in pigs using zinc-finger nucleases. Proc Natl Acad Sci U S A. 2011; 108: 12013-7. doi:10.1073/pnas.1106422108.

17. Carlson DF, Tan W, Lillico SG, Stverakova D, Proudfoot C, Christian M, et al. Efficient TALEN-mediated gene knockout in livestock. Proc Natl Acad Sci U S A. 2012; 109: 17382-7. doi:10.1073/pnas.1211446109.

18. He J, Wang Q, Ye J, Hu X, Li N. Identification of porcine polycystic kidney disease 1 (PKD1) gene: molecular cloning, expression profile, and implication in disease model. Gene. 2011; 490: 37-46. doi:10.1016/j.gene.2011.08.027.

19. Wang $\mathrm{Q}$, Yin $\mathrm{H}, \mathrm{He} J$, Ye J, Ding F, Wang $\mathrm{S}$, et al. cDNA cloning of porcine PKD2 gene and RNA interference in LLC-PK1 cells. Gene. 2011; 476: 38-45. doi:10.1016/j.gene.2011.01.017.

20. He J, Ye J, Li Q, Feng Y, Bai X, Chen X, et al. Construction of a transgenic pig model overexpressing polycystic kidney disease 2 (PKD2) gene. Transgenic Res. 2013; 22: 861-7. doi:10.1007/s11248-012-9686-z.

21. Yu Shu-min, Wang Chuan-wu, Zhao De-ming, Zhang Qing-cai, De-zhi P. Raising and pathogen purification of chinese experimental mini-pig. Laboratory Animal Sciences and Management. 2003; 20: 44-6.

22. Wei H, Li Q, Li J, Li Y, Dai Y, Ma Y, et al. Effect of leptin on oocyte maturation and subsequent pregnancy rate of cloned embryos reconstructed by somatic cell nuclear transfer in pigs. Progress in Natural Science. 2008; 18: 1583-7. doi:10.1016/j.pnsc.2008.05.018.

23. Groenen MA, Archibald AL, Uenishi H, Tuggle CK, Takeuchi Y, Rothschild MF, et al. Analyses of pig genomes provide insight into porcine demography and evolution. Nature. 2012; 491: 393-8. doi:10.1038/nature11622.

24. Garcia-Gonzalez MA, Outeda P, Zhou Q, Zhou F, Menezes LF, Qian F, et al. $\mathrm{Pkd} 1$ and $\mathrm{Pkd} 2$ are required for normal placental development. PLoS One. 2010; 5. doi:10.1371/journal.pone.0012821. 
25. Pei Y, Obaji J, Dupuis A, Paterson AD, Magistroni R, Dicks E, et al. Unified criteria for ultrasonographic diagnosis of ADPKD. J Am Soc Nephrol. 2009; 20 : 205-12. doi:10.1681/ASN.2008050507.

26. Bae KT, Zhu F, Chapman AB, Torres VE, Grantham JJ, Guay-Woodford LM, et al. Magnetic resonance imaging evaluation of hepatic cysts in early autosomal-dominant polycystic kidney disease: the Consortium for Radiologic Imaging Studies of Polycystic Kidney Disease cohort. Clin J Am Soc Nephrol. 2006; 1: 64-9. doi:10.2215/CJN.00080605.

27. Grantham JJ, Chapman AB, Torres VE. Volume progression in autosomal dominant polycystic kidney disease: the major factor determining clinical outcomes. Clin J Am Soc Nephrol. 2006; 1: 148-57. doi:10.2215/CJN.00330705.

28. Hopp K, Ward CJ, Hommerding CJ, Nasr SH, Tuan HF, Gainullin VG, et al. Functional polycystin-1 dosage governs autosomal dominant polycystic kidney disease severity. J Clin Invest. 2012 · 122 : 4257-73 doi:10.1172/JCI64313.

29. Norman J. Fibrosis and progression of autosomal dominant polycystic kidney disease (ADPKD). Biochim Biophys Acta. 2011; 1812: 1327-36. doi:10.1016/j.bbadis.2011.06.012.
30. Walz G, Budde $K$, Mannaa $M$, Nurnberger J, Wanner $C$, Sommerer $C$, et al. Everolimus in patients with autosomal dominant polycystic kidney disease. $\mathrm{N}$ Engl J Med. 2010; 363: 830-40. doi:10.1056/NEJMoa1003491.

31. Serra AL, Poster D, Kistler AD, Krauer F, Raina S, Young J, et al. Sirolimus and kidney growth in autosomal dominant polycystic kidney disease. N Engl J Med. 2010; 363: 820-9. doi:10.1056/NEJMoa0907419.

32. Ibrahim Z, Busch I, Awwad M, Wagner R, Wells K, Cooper DK. Selected physiologic compatibilities and incompatibilities between human and porcine organ systems. Xenotransplantation. 2006; 13: 488-99. doi:10.1111/j.1399-3089.2006.00346.x.

33. Ahrabi AK, Terryn S, Valenti G, Caron N, Serradeil-Le Gal C, Raufaste D, et al. PKD1 haploinsufficiency causes a syndrome of inappropriate antidiuresis in mice. J Am Soc Nephrol. 2007; 18: 1740-53. doi:10.1681/ASN.2006010052.

34. Bastos AP, Piontek K, Silva AM, Martini D, Menezes LF, Fonseca JM, et al. Pkd1 haploinsufficiency increases renal damage and induces microcyst formation following ischemia/reperfusion. J Am Soc Nephrol. 2009; 20: 2389-402. doi:10.1681/ASN.2008040435. 